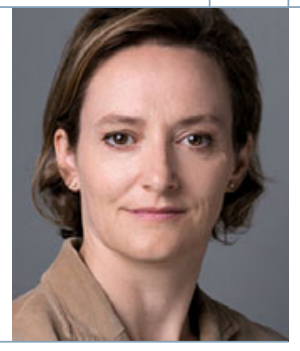

\title{
Chance verpasst: ein rückwärtsgewandtes Urteil des EuGH zu Genom-editierten Organismen
}

DOI: $10.1007 / \mathrm{s} 12268-018-0959-9$

(C) Springer-Verlag 2018

Die Mehrheit der Wissenschaftler hat mit großer Enttäuschung auf das Urteil des Europäischen Gerichtshofs (EuGH) vom 25. Juli 2018 zu Genom-editierten Organismen [1] reagiert. Laut diesem Urteil sind Organismen auch dann als gentechnisch verändert (GVO) zu betrachten, wenn sie durch „neue Mutagenese-Verfahren“ (z. B. CRISPR/ Cas oder andere Genomeditierungstechnologien) entstanden sind, aber nur Punktmutationen oder kleine Insertionen/Deletionen enthalten. Damit folgt das Gericht weiterhin dem Prinzip der Beurteilung des „Prozesses“, anstelle des „Produktes“.

Diese Ansicht ist diametral entgegengesetzt zur Meinung der meisten Forscher und Wissenschaftsorganisationen [2, 3]. Genetiker werden einwenden, dass Genom-editierte Organismen technisch nicht unterscheidbar sind von Organismen, die durch Zufallsmutagenese, induziert oder nicht, generiert wurden. Somit ist es widersinnig und wissenschaftlich nicht haltbar, hier eine Unterscheidung aufrecht zu erhalten, die im Zweifelsfall nicht überprüft werden kann.

Das EuGH-Urteil wird klare negative Auswirkungen auf Forschung und Entwicklung in Europa haben. Die europäischen Länder werden sich schwertun mit einer Zulassung von Genom-edierten Pflanzen für die Landwirtschaft, da sie zukünftig wie GV-Pflanzen behandelt werden [4]. Dabei wäre gerade hier eine zukunftsorientierte Perspektive wünschenswert, um ökologisch verträglichere und klimatisch besser angepasste Zuchtsorten schnell bereitstellen zu können, damit Lebens- und Futtermittelsicherheit auch in Zeiten rapider klimatischer Veränderungen gewährleistet sind.

Einmal mehr hat hier nicht die Vernunft, sondern die europäische Übervorsicht gewonnen, die durch die reißerische Stimmungsmache einiger Nichtregierungsorganisationen (NGOs) befeuert wird. Dass diese Aktionen dem finanziellen Eigennutzen und der eigenen Legitimation dienen, scheinen die meisten Bürger nicht zu realisieren. Die NGOs verquicken in ihrer Argumentation gesundheitliche Risiken für den Konsumenten mit politischen Argumenten gegen Großkonzerne und deren wirtschaftliche Übermacht und lassen den Verbraucher in der irrigen Meinung, dass der Verzehr von „Genfood“ gesundheitsschädlich wäre. Ironischerweise befördern solche NGOs durch ihre sensationsgetriebene Vorgehensweise der Verteufelung der Gentechnik den Umstand, dass nur Großkonzerne die Millionen schweren Zulassungsverfahren finanzieren können - kleinere Saatgutzüchter haben keine Chance.

Oder es wird vorgegaukelt, dass die Risiken unkalkulierbar seien. Dabei ist deren Anwendung schon lange Routine: in anderen Regionen der Welt, v. a. in den USA, Brasilien, Argentinien und China, werden GVPflanzen (Soja, Mais) seit Jahren im großen Stil angepflanzt [5]. Kein einziger Fall einer Gesundheitsschädigung durch gentechnische Veränderung beim Verzehr von GVOs ist bekannt. Man kann also guten Gewissens auch die derzeitig genutzten GV-Pflanzen mit ihren Transgenen, die tatsächlich Fremdgenen entsprechen, als gesundheitlich sicher betrachten. Im gleichen Atemzug sollte nicht unerwähnt bleiben, dass auch durch jahrzehntelange Nutzung von Gentechnik-Methoden in Laboratorien weltweit noch kein Wissenschaftler zu Schaden gekommen ist, man also auch hier getrost eine Deregulierung vornehmen könnte.

Dabei hat das EuGH-Urteil nicht nur Auswirkungen auf die landwirtschaftliche Nutzung von Genomeditierung. Was ist mit der medizinischen Nutzung in der Gentherapie, oder der Herstellung von Medikamenten? Müssten dann Patienten, die Genom-editierte Versionen körpereigener Zellen tragen, als GVOs deklariert werden? In medizinischen Fragen, wo der persönliche Nutzen klar erkennbar ist, ist die Bevölkerung eher gewillt, dem Einsatz von Gentechnik zuzustimmen.

Vielleicht besteht der bisherige Fehler der Entwicklung von GVOs tatsächlich darin, dass der Konsument den eigenen Vorteil nicht klar erkennen kann. Was wäre, wenn die Genomeditierte Tomate billiger wäre als die konventionell gezüchtete? Wenn sie auch noch schöner rot, oder bunt, nicht matschig sondern wohlschmeckender wäre? Würde der Normalbürger dann nach Gefühl (Angst vor Gentechnik) oder nach Geldbeutel entscheiden?

Auf jeden Fall ist der Einsatz von Genomeditierung nicht aufzuhalten. Wenn Vorteile da sind, werden sich die Methode und auch die Produkte auf dem Markt durchsetzen. Bloß werden wir nicht selber an ihrer Entwicklung beteiligt sein, sondern als Zaungäste darauf warten, dass sie aus den USA bei uns ankommen. Für Forschung und Innovation setzt dies kein positives Zeichen: Einmal mehr steht Europa hintan.

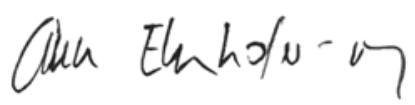

Ann Ehrenhofer-Murray

Präsidentin der Gesellschaft für Genetik (GfG)

Korrespondenzadresse:

Prof. Dr. Ann Ehrenhofer-Murray

Humboldt-Universität zu Berlin

Philippstraße 13, Rhoda-Erdmann-Haus

D-10099 Berlin

Tel.: 030-2093-49630

ann.ehrenhofer-murray@hu-berlin.de

\section{Literatur}

[1] http://curia.europa.eu, Aktenzeichen C-528/16 [2] Nationale Akademie der Wissenschaften Leopoldina, Deutsche Forschungsgemeinschaft, acatech - Deutsche Akademie der Technikwissenschaften, Union der deutschen Akademien der Wissenschaften (2015) Chancen und Grenzen des genome editing, Halle (Saale),

www.dfg.de/download/pdf/dfg_im_profil/reden_stellungnah men/2015/stellungnahme genome editing 2015.pdf [3] Nationale Akademie der Wissenschaften Leopoldina (2017) Ethische und rechtliche Beurteilung des genome editing in der Forschung an humanen Zellen,

www.leopoldina.org/uploads/tx_leopublication/2017_Diskuss ionspapier_GenomeEditing.pdf

[4] Wolt JD (2017) Safety, Security, and Policy Considerations for Plant Genome Editing. Prog Mol Biol Transl Sci 149:215241

[5] Informationsplattform zur Gentechnik im Agrar- und Lebensmittelbereich, www.biotech-gm-food.com 